\title{
ORIGINAL ARTICLE \\ Effect of Abdominal Physiotherapy in Reduction of Inter Recti Distance in Postpartum Females
}

\author{
KHIZRA KAZMI ${ }^{1}$, GULL MAHNOOR HASHMI ${ }^{2}$, MUHAMMAD BILAL $^{3}$, NASIR IQBAL $^{4}$, WAJEEHA ${ }^{5}$, AHSAN ILYAS $^{6}$, ANUM $^{2}$ \\ ASIF $^{7}$, JOHAM SHAFIQ $^{8}$ \\ ${ }_{1,2,5-7}$ Physiotherapist \\ ${ }^{2}$ Assistant Professor Orthopedics, Al-Aleem Medical College/ Gulab Devi Teaching Hospital Lahore \\ ${ }^{3}$ Associate Professor Azra Naheed Medical College \\ Correspondence to: Dr. Gull Mahnoor Hashmi, Email: mahnoor646@gmail.com, Cell: +92-321-4177904
}

\begin{abstract}
Aim: To see the effect of abdominal exercises in the recovery of diastasis recti in post natal females.

Methodology: A Quasi Experimental study design was used to enroll 40 participants from OPD of Gynecology and Obstetrics from a Tertiary Care Hospital Lahore between ages ranged from 18 to 30 years. Using purposive sampling all postpartum females having diastasis of rectus abdominis muscles with inter recti distance more than 2 fingers width were enrolled. All the study participants received abdominal muscle exercises program for 30 min a day in 8 week program. inter recti distance was measured by using two finger palpation method. All the pre intervention and post intervention data were recorded into a short structured proforma. Results were considered significant for $p$ value $\leq 0.05$.

Results: 40 participants were selected in this study with an age from 18- 30 years. The inter recti distance was significantly reduced as $p$ value was $\leq 0.05$ for both (above umbilicus and below umbilicus) groups.

Conclusion: It was observed that abdominal exercises helped in the recovery of diastasis and reduced the separation between the muscles in postpartum women. It also enlightens the importance of the prescription of physiotherapy in reduction of post partum complications.

Keywords: Abdominal Exercises, Diastasis Recti, Pregnancy, Postnatal period,
\end{abstract}

\section{INTRODUCTION}

One of the most eminent phase of women's life is pregnancy. ${ }^{1}$ Although pregnancy is a natural physiological process but postnatal complication poses a unique and gender based challenge for physical therapist. ${ }^{2} \mathrm{~A}$ pregnant female undergoes a number of anatomical changes which includes increase in the size of uterus by 5 to 6 times. Once the uterus is enlarged it is becomes an abdominal organ. The connective tissues of pelvis are fibro elastic in nature and to support the uterus they are stretched to the maximum limit. 2,3 Rectus abdominis is an abdominal muscle which is stretched to its last limit and the capacity to generate abdominal contraction is reduced by the end of pregnancy. ${ }^{2}$ Right after the delivery the body of the new mother begin to return in its normal shape and function. She may be thrilled by the fitness of her abdomen after few hours of delivery. Most common complication that occurs after delivery is diastasis of rectus abdominis muscle. ${ }^{4}$ Diastasis recti is condition defined as a palpable midline gap of more than $2.5 \mathrm{~cm}$ or any visible bulging on exertion or any separation more than 2 finger width below or above the level of umbilicus. . $^{1,2,4,5}$

Major factors that cause this complication are increased amount of Relaxin, Progesterone and Estrogen that softens the integrity of muscles. ${ }^{6,7}$ The post natal women perceive no pain at rest but a bulge and core instability combined with discomfort and pain on activity. multiparous women are the most potential victim of diastasis recti because the abdominal wall have been stretched multiple times ${ }^{2}$.

Received on 24-02-2021

Accepted on 12-06-2021
Boissonnault and Blaschak conducted multiple descriptive studies in which they found diastasis is most common in the third trimester and in one study they concluded that the separation between the muscles continued between 5 weeks to 3 months postpartum. ${ }^{2,5}$ Decrease in musculature of abdominal muscles diminish the mechanical control of abdomen and cause physical discomfort such as back pain, limiting physical activity such as bending and flexiuon of trunk. 2,4,6,8 Diastasis recti can be treated conservatively and in extreme cases surgically. ${ }^{2}$ Expecting a pre pregnancy shape shortly after delivery is unrealistic. Multiple treatments which include bracing, faradic stimulation, back care lifting, tupler technique may help to conserve the structure of rectus abdominis. ${ }^{1,9-11}$ Hammer and Hinterman published an article in 1998 which showed the importance of exercise in aiding weight loss and stress management. ${ }^{10}$

\section{MATERIALS AND METHODS}

A Quasi experimental study design was used to enroll 40 participants from OPD of Gynecology and Obstetrics from a tertiary care hospital Lahore.

Sample Selection: All postpartum females having diastasis of rectus abdominis muscles with inter recti distance more than 2 fingers width were enrolled. Females with caesarean section, recent abdominal surgery and/or presence of any condition associated with pregnancy like polyhydramnios, fetal microsomia, hypertension induced by pregnancy were omitted.

Data Collection: An informed consent was taken after the illustration of the study and procedure from all participants. Then the subjects received abdominal muscle exercises program for 30min/day, 5 times/ week for period of 8 
weeks. Diastasis recti palpation method was performed to check for Diastasis recti and inter recti distance. All the relevant data were recorded into a short structured proforma

Procedure: A slight head lift in crook lying will require a rectus abdominis contraction, and will allow for assessment of the diastasis recti abdominis. A small separation of the midline at the abdominals, approximately one to two fingers' width, is common after most pregnancies, and is not a problem. But if the gap at the midline is:

- More than $21 / 2$ finger widths

- does not shrink as the patient tighten her abdominals

or

- a small mound protruding at her midline

The procedure was repeated thrice on a single patient and patient was allowed to lower down to normal position. ${ }^{1,2}$ subjects were allowed to rest between measurements and were allowed to rest if she feels uncomfortable or fatigued.

Intervention: Conservative management, such as specific therapeutic exercises, core contraction, seated squeeze, squat with squeeze or push up directed by a physiotherapist, or health care professional wellacquainted with diastasis recti abdominis, is usually the first line of intervention..

Data Analysis: Two finger palpation method was used to obtain pre and post intervention data. Data was analyzed using spss v.26. All outcomes were expressed in terms of mean and standard deviation. Data within each group was compared using test of significance.

\section{RESULTS}

The mean age of study participants was $27.3 \pm 4.5$ years with minimum age of 18 years and maximum age of 30 years.

Table 1: Socio-demographic Profile of participants

\begin{tabular}{|l|c|}
\hline Age mean \pm S.D (years) & $27.3 \pm 4.5$ \\
\hline BMI mean \pm S.D $\left(\mathrm{kg} / \mathrm{m}^{2}\right)$ & $23.1 \pm 4.4$ \\
\hline Parity $\mathrm{n}(\%)$ & \\
Primiparous & $23(57.5 \%)$ \\
Multiparous & $17(42.5 \%)$ \\
\hline
\end{tabular}

Mean inter recti gap after two finger palpation method pre intervention was $2.36 \pm 0.94 \mathrm{~cm}$, post intervention was $0.88 \pm 0.75 \mathrm{~cm}$. Mean inter recti distance at below umbilicus pre intervention was $2.06 \pm 0.76 \mathrm{~cm}$, post intervention was $0.75 \pm 0.36 \mathrm{~cm}$. The inter recti distance was significantly reduced as $p$ value was $\leq 0.05$ for both groups.

Table 2: Comparison of Pre and Post intervention outcome after one finger palpation technique

\begin{tabular}{|l|c|c|}
\hline & Pre intervention & Post intervention \\
\cline { 2 - 3 } & Mean \pm S.D & Mean \pm SD \\
\hline Above Umbilicus $(\mathrm{cm})$ & $2.36 \pm 0.94$ & $0.88 \pm 0.75$ \\
\hline Below Umbilicus $(\mathrm{cm})$ & $2.06 \pm 0.76$ & $0.75 \pm 0.36$ \\
\hline
\end{tabular}

\section{DISCUSSION}

In this study we used two finger technique to evaluate the inter rectus difference. Keeler and albert conducted a study in 2012 and showed that two finger technique is the most widely used technique in the diagnosis of diastasis recti. ${ }^{12}$ While studying the inter rectus difference Hernandz discussed in his study the functional limitation that comes along with this inter rectus difference. ${ }^{9}$

To regain muscle strength the prescribed exercises earlier in this study will help in hypertrophy of muscles, recruitment of more motor units and improves functional stability. The change and improvement in muscular structure and increased metabolic demand was studied by Eldeeb in 2013. ${ }^{13}$

The exercises prescribed for the treatment of diastasis recti increases the core stability and improve range of motion in back and support weight lifting activities. Therefore itis very important regain strength and return to postpartum shape by exercising regularly after delivery. ${ }^{7}$ Most of the abdominal exercises should be incorporated with respiratory exercises because exhalation will increase intra abdominal pressure and reduce inter recti distance. ${ }^{14}$ Worren et al and Ivaniac described in their recent study that incorporation of exhalation along with abdominal exercises not only increased muscle strength but assisted in reducing diastasis recti. ${ }^{13}$ The concept of Reduction in inter recti distance by isometric exercises was further enforced by the study done by A G pascol. ${ }^{9}$

Our study is further explained by evidence stated in study by Amel M yusuf where abdominal exercises did not increase the pelvic tilt and in another study by Wohlfart in which he examined and proved that straight leg raise ad slow lifting of legs can increase abdominal strength without any harmful effect. ${ }^{15}$ This study has primarily concentrated on the reduction of diastasis recti through abdominal exercises.

In addition to that specifically tranversus abdominus vertical stretch can lead to optimum management of reduction in muscular gap. ${ }^{7}$ However the other study shows that there is no certain evidence that the vertical tension is created by rectus abdominis or the group of abdominal muscles that pull together to reduce the inter recti distance. ${ }^{9,16}$

Proper administration of exercises is very important as stated in the study conducted by Opalabezdick. He concluded the shortly after delivery abdominal exercises should be administered but with increasing intensity and duration until diastasis is recovered. ${ }^{14}$

In this current study there is a significant improvement in the reduction of inter recti gap and it helped in regaining pre pregnancy muscle function.

\section{CONCLUSION}

Abdominal exercises are found to be very effective in reduction of inter recti distance. Not only this it helps in strengthening of abdominal muscles and the results of the study support prescription of abdominal exercise program during the postpartum period.

Implications to practice: All participants showed reduction in the inter recti gap after eight week of intervention and hencemaking it eminent to inculcate abdominal physiotherapy in postpartum physiotherapy plan.

Limitations of the study: Although the findings of the study were significant but there are few limitations that 
needs to be addressed. The limitations of the study were small sample size and the effect of body weight in development of diastasis was not considered.

Recommendations: larger sample size should be considered for further studies and the prescription of abdominal exercise should be made compulsory for post natal women to avoid diastasis of muscles keeping in view the underlying conditions.

\section{REFERENCES}

1. Acharry N, Kutty RK. Abdominal exercise with bracing, a therapeutic efficacy in reducing diastasis-recti among postpartal females. Int J Physiother Res. 2015;3(2):9991005.

2. Khandale SR, Hande D. Effects of abdominal exercises on reduction of diastasis recti in postnatal women. IJHSR. 2016;6(6):182-91.

3. Sapsford R, Bullock-Saxton J, Markwell S. Women's health: a textbook for physiotherapists: WB Saunders; 1998.

4. Brook G, Coldron Y, Evans G, Gulliford G, Haslam J, Hawkes R, et al. Physiotherapy in women's health. Tidy's Physiotherapy. 2008:113-44.

5. Boissonnault JS, Blaschak MJ. Incidence of diastasis recti abdominis during the childbearing year. Physical therapy. 1988;68(7):1082-6.

6. Chiarello CM, Falzone LA, McCaslin KE, Patel MN, Ulery $K R$. The effects of an exercise program on diastasis recti abdominis in pregnant women. Journal of Women's Health Physical Therapy. 2005;29(1):11-6.

7. Parker MA, Millar LA, Dugan SA. Diastasis rectus abdominis and lumbo-pelvic pain and dysfunction-are they related? Journal of women's health physical therapy. 2009;33(2):1522.

8. Pivarnik JM, Chambliss HO, Clapp JF, Dugan SA, Hatch MC, Lovelady CA, et al. Impact of physical activity during pregnancy and postpartum on chronic disease risk. Medicine \& Science in Sports \& Exercise. 2006;38(5):989-1006.

9. Pascoal A, Dionisio S, Cordeiro F, Mota P. Inter-rectus distance in postpartum women can be reduced by isometric contraction of the abdominal muscles: a preliminary casecontrol study. Physiotherapy. 2014;100(4):344-8.

10. Hammer RL, Perkins J, Parr R. Exercise during the childbearing year. The journal of perinatal education. 2000;9(1):1-13.

11. Eggen $M H$, Stuge B, Mowinckel $P$, Jensen KS, Hagen KB. Can supervised group exercises including ergonomic advice reduce the prevalence and severity of low back pain and pelvic girdle pain in pregnancy? A randomized controlled trial. Physical therapy. 2012;92(6):781-90.

12. Keeler J, Albrecht M, Eberhardt L, Horn L, Donnelly C, Lowe D. Diastasis recti abdominis: a survey of women's health specialists for current physical therapy clinical practice for postpartum women. Journal of women's health physical therapy. 2012;36(3):131-42.

13. El-Mekawy HS, Eldeeb AM, El-Lythy MA, El-Begawy AF, editors. Effect of abdominal exercises versus abdominal supporting belt on post-partum abdominal efficiency and rectus separation. Proceedings of World Academy of Science, Engineering and Technology; 2013: World Academy of Science, Engineering and Technology (WASET).

14. Opala-Berdzik A, Dabrowski S. Physiotherapy in diastasis of the rectus muscles of abdomen in women during pregnancy and postpartum. Physiotherapy Quarterly. 2009;17(4):67.

15. Chiarello CM, McAuley JA. Concurrent validity of calipers and ultrasound imaging to measure interrecti distance. journal of orthopaedic \& sports physical therapy. 2013;43(7):495-503.

16. Amalia R, Masita ED, editors. Implementation of Degread Examination Asseted By Abdominalis Certification in Paper Mother. PROCEEDING SURABAYA INTERNATIONAL HEALTH CONFERENCE 2019; 2019. 